\title{
The Research of Capacitor Voltage Balancing Strategy of MMC
}

\author{
Xiufang Jia, Jichen Liu, Jianpo Zhang \\ Department of Power Engineering, North China Electric Power University,Baoding,071000, China
}

\begin{abstract}
Compared with the two level voltage source, the modular multilevel converter (MMC) adopting the modular design dose not have the disadvantages such as the direct series connected Insulated gate bipolar (IGBT),high generation of harmonics, etc. MMC is currently studied and applied in high voltage and high power conversion applications. But the main problem in the new potential MMC topology is the voltage balance of the capacitors of their sub modules to protect the capacitors voltage fluctuation within the same voltage levels, so the voltage balance strategy plays an important role in improve it's performance. In order to achieve voltage balance, this paper ameliorates the traditional balance arithmetic through changing the charge and uncharged time; what is more, a capacitance voltage diversity index(CVDI) is defined firstly to adjust the sorting frequency, so the switching frequency of IGBT is reduced under satisfying the balance demand. At last, the simulation is carried out to verify these algorithms validity.
\end{abstract}

Keywords- Modular multilevel Converter; voltage balance; sort sequence balance; capacitance voltage diversity index.

\section{INTRODUCTION}

Modular multi-level converter (MMC), as a new topology for high voltage power conversion, which through a series of sub-module, constitute each phase inverter bridge arm. The modular construction makes it more scalable[1,2].

However, the capacitors used as energy storage element are distributed in sub-module, so the capacitor charging and discharging time, loss and parameters of the different sub-modules will result in differences in the capacitor voltage, so that imbalance occurs between the capacitor voltage of the module. It is necessary to consider capacitor voltage balance control for modular multi-level inverters.

For the balancing strategy based on the sort of capacitor voltage module, if appropriately increase in ordering frequency, capacitance voltage balancing effect will be better. This correspondingly increases the computational spending of switching losses and the system, on the contrary, the lower the frequency the equalizing effect of ordering the worse, and even leads to system instability. The choice of ordering frequency is critical.

\section{THE MMC CIRCUIT MODEL}

Figure 1 shows the equivalent circuit of MMC system, $\mathrm{R}$ is for equivalent loss resistance of the bridge arm device,and $L$ is the equivalent inductance of the transformer and bridge arm.The upper and lower bridge arm module voltage can be equivalent to a voltage controlled voltage source.P and $\mathrm{N}$ represent positive and negative bus of DC,and their potential are Udc/2 and $\mathrm{Udc} / 2$ with respect to the reference neutral point $\mathrm{O}$.

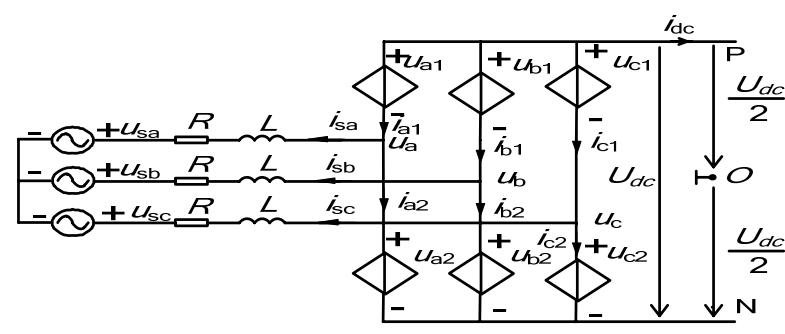

Figure.1 The topology circuit of MMC

\section{The MMC VOLTAGE BALANCING StRATEGy RESEARCH}

Under the action of the module drive signal, capacitor voltage converts work state over and over between charge, discharge, and cut state. According to the relationship between the capacitor voltage and current, the capacitor voltage can be expressed as Formula (1).

$$
u_{c}(t)=u_{c}\left(t_{0}\right)+\frac{1}{c} \int_{t_{0}}^{t} i_{c}(\tau) d \tau
$$

As can be seen from Equation 1,the influencing factors of capacitor voltage include the initial capacitor voltage value uc(t0),modules capacitance parameters $\mathrm{C}$, charging current $\mathrm{ic}(\mathrm{t})$ and charging time t-t0.In practice, because of the differences of the initial value of the capacitor voltage and the capacitance parameter, with the bridge arm current changing over time, it is hard to control them.

Therefore, it can adjust the time of module capacitance in the three states (charging, discharge, excision) to achieve the purpose of the capacitance voltage adjustment. It is based on this principle to form a capacitor voltage sorting method, due to the traditional sort worked in a constant sort frequency, so this is defined as a constant frequency sort (Constant frequency sort, CFS).

For voltage balancing strategy, the primary goal is to ensure a balanced control between the module voltage, followed should be possible to reduce the switching frequency. This paper defines an adaptive frequency sorting algorithms according to Voltage balancing degree (Variable frequency sort, VFS).

In order to characterize the balance degree of the module capacitor voltage, this paper introduces the 
Standard Deviation. First, it defines each bridge arm module capacitor voltage as a data set,as shown in Formula (2).

$u_{\Phi_{-} m_{-} c}=\left\{u_{\Phi_{-} m_{-} c_{-} 1}, u_{\Phi_{-} m_{-} c_{-} 2} \ldots, u_{\Phi_{-} m_{-} c_{-} n}\right\}$

Which

phase

$\phi \in\left(P \text { hase } e_{-} a, P \text { hase } e_{-} b, P \text { hase } e_{-}\right)_{\text {, br }}$

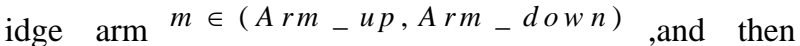
comes to the mean square error, namely difference degree, of the bridge arm capacitor voltage as shown in formula (3), where $n$ is the number of modules.

$$
\delta\left(u_{c}\right)=\sqrt{\frac{1}{n} \sum_{k=1}^{n}\left(u_{\Phi_{-} m_{-} c_{-} k}-u_{e}\right)^{2}}
$$

Deviation rate of the capacitor voltage is defined by formula (4).

$$
\Delta u \%=\left(u_{c}-u_{e}\right) \times 100 / u_{e}
$$

In practice, in order to ensure the normal operation of the MMC, it is usually to ensure that the module capacitor voltage deviation rate can not exceed 5\%[14].

Formula (5) is obtained by calculating at formula (3) in per-unit value normalization, then the difference degree of module voltage can be figured out. The larger the degree of difference between the capacitor voltage module is, indicating that the higher the voltage imbalance between modules degree.

$$
\delta\left(u_{c}\right)=\sqrt{\frac{1}{n} \sum_{k=1}^{n}\left(\frac{u_{\Phi_{-} m_{-} c_{-} k}-u_{e}}{u_{e}}\right)^{2}} \leq 5 \%
$$

The system automatically adjusts the sort frequency according to the capacitor voltage difference degrees, when the bridge arm capacitor voltage difference is greater than the set value, it indicates that the voltage is relatively large degree of dispersion, at this time it should be to reorder the capacitor voltage of bridge arm module and adjust the order of module conduction to change the module charging and discharging time. When the difference is less than the set, it indicates that the bridge arm module voltage differences from each other is not great, basically at the same level, then it should maintain the original sorting result, keeping the switch state of module unchanged, thereby reducing switching losses.

\section{SIMULATION ANALYSIS}

In order to verify the balancing effect of the proposed variable frequency capacitor voltage, build a model in PSCAD/EMTDC for the simulation analysis. Valid values for the AC line voltage is $220 \mathrm{kV}$, with converter transformer ratio $220 / 210 \mathrm{kV}$,commutation reactance $0.1 \mathrm{pu}$,rated DC voltage Udc $=400 \mathrm{kV}$.Inverter constituted by the six-arm, each arm has 20 sub module, with the level number 21.Sub-module capacitance value is
3mF,arm inductance $40 \mathrm{mH}$,using fixed-active and fixed-reactive power control strategy.

The minimum balance frequency is $2000 \mathrm{~Hz}$, the capacitor voltage difference of the bridge arm to be $1 \%$,Figure 2 shows the waveform of simulation results.

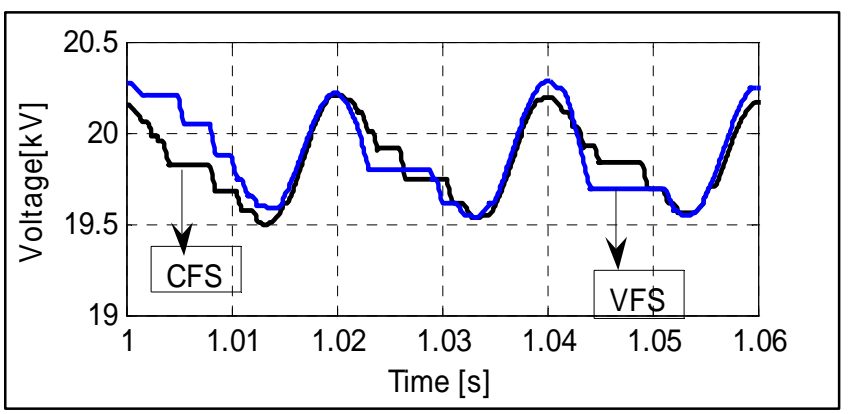

Fig.2.bridge arm sub-module voltage

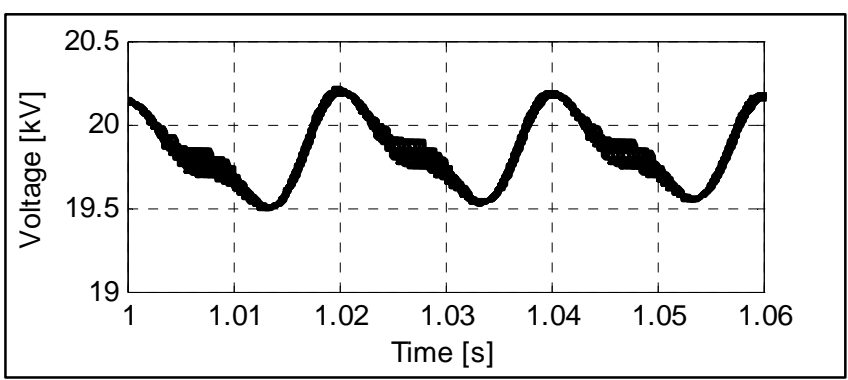

Fig.3.bridge arm module voltage under the fixed-frequency

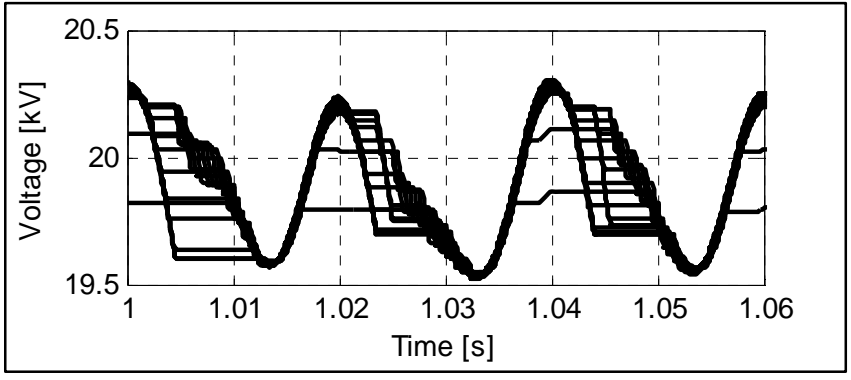

Fig.4.bridge arm module voltage under the variable frequency

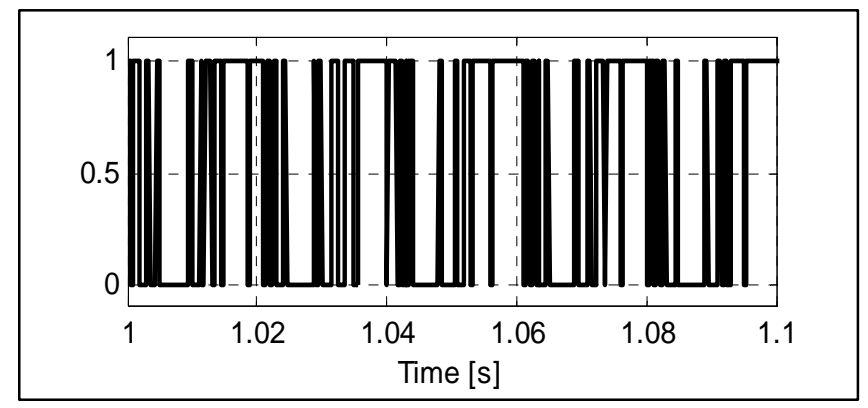

Fig.5.trigger pulse of the fixed-frequency bridge arm module 


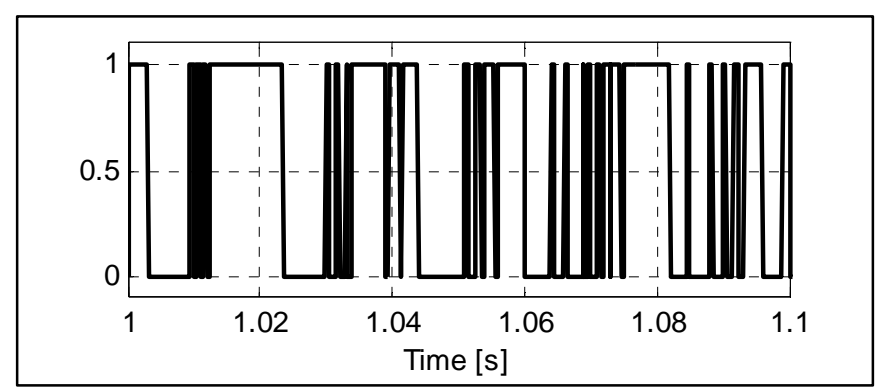

Fig.6.trigger pulse of the variable frequency bridge arm module

Through simulation analysis described above, VFS balance under the condition of meeting the capacitor voltage difference degree, which is to ensure overall balanced capacitor voltage ,reduce the module switching frequency and reduce the effect of the switching loss.

\section{V.CONCLUSION}

This paper analyzes the affecting factors about the sub-module capacitor voltage difference in the MMC, pointing out that the balance control of capacitor voltage can be achieved by adjusting the sub-module capacitor charging and discharging time. According to the characteristics of standard deviation that can reflect the dispersion degree of data, apply it to the capacitor voltage balance as a quantitative indicator to reflect the dispersion degree of bridge arm module capacitor voltage between each other. In consideration of the limitations of traditional voltage balance control algorithm, this paper proposes an adaptive frequency sorting algorithm, under the condition of ensuring the overall balance of the capacitor voltage, which try to avoid switching module status and reduce the switching frequency.

\section{REFERENCES}

[1] $\mathrm{Xu}$ Zheng, Chen Hairong. Review and applications of VSC HVDC[J]. High Voltage Engineering, 2007, 33(1): 1-10(in Chinese).

[2] Li Gengyin, Lu Pengfei, Li Guangkai etal. Development and prospects for HVDC Light[J]. Automation of Electric Power System, 2003, 27(4): 1-5.

[3] Yang Xiaofeng, ZHENG qionglin. A Novel Universal Circulating Current Suppressing Strategy Based on the MMC Circulating Current Model [J]. Proceedings of the CSEE, 2012, 32(18): 59-65(in Chinese).

[4] DING Guan-jun, DING Ming, TANG Guang-fu, HE Zhi-yuan. Submodule Capacitance Parameter and Voltage Balancing Scheme of a New Multilevel VSC Modular [J]. Proceedings of the CSEE, 2009, 29(30): 1-6(in Chinese).

[5] Liu Zhongqi, Song Qiang, Liu Wenhua. VSC-HVDC system based on modular multilevel converters[J]. Automation of Electric Power System, 2010, 34(2): 53-58(in Chinese).

[6] Guan Minyuan, Xu Zheng, Tu Qingrui, et al. Nearest level modulation for modular multilevel converters in HVDC transmission[J] . Automation of Electric Power System , 2010 , 34(2): 48-52(in Chinese).

[7] Gemmell B, Dorn J, Retzmann D, et al. Prospects of multilevel
VSC technologies for power transmission[C]//IEEE PES Transmission and Distribution Conference and Exposition. Chicago, USA: IEEE, 2008: 1-16.

[8] Ding Guanjun, Tang Guangfu, Ding Ming, et al. Topology mechanism and modulation scheme of a new multilevel voltage source converter[J]. Proceedings of the CSEE, 2009, 29(36): 1-8.

[9] FRANQUELOLG, RODRIGUEZJ, LEONJI, etal. The age of multi level converters arrives. IEEE Industrial Electronics Magazine, 2008, 2(2): 28-39.

[10] PEREZM, RODRIGUEZJ, PONTJ, etal. Power distribution in hybrid multi cell converter with nearest level modulation / / Proceedings of IE EE International Symposium on industrial Electronics, June 4-7, 2007, Vigo, Spain: 736-741.

[11] M. Dommaschk, "Drive for a phase module branch of a multilevel converter,”Int. Patent WO2008/086760A1, Jul. 24, 2008.

[12] Qingrui $\mathrm{Tu}$ and Zheng Xu, "Reduced Switching-Frequency Modulation and Circulating Current Suppression for Modular Multilevel Converters,” IEEE Trans. Power Del., vol. 26, no. 3, pp. 2009-2019, Jul. 2011.

[13] GUAN Minyuan, Xu Zheng. Optimized Capacitor Voltage Balancing Control for Modular Multilevel Converter Based VSC-HVDC System [J]. Proceedings of the CSEE, 2011, 31(12): 6-11(in Chinese).

[14] Tang Guangfu. Based on voltage source inverter of high-voltage direct current transmission technology[M]. Beijing: China power press,2010(in Chinese). 